\title{
Identifying Risk Factors for Regional Recurrence in Early-Stage Breast Cancer with PTI-2 and Negative Sentinel Lymph Node Biopsy
}

This article was published in the following Dove Press journal: Cancer Management and Research

\author{
Kai-yun You ${ }^{1-3}$ \\ Zhuo-fei Bi $\mathbb{D D}^{1-3}$ \\ Lin Ding ${ }^{1-3}$ \\ Yu-jia $\mathrm{Ma}^{3}$ \\ Yi-min Liu ${ }^{1,3}$ \\ He-rui Yao ${ }^{1,2,4,5}$ \\ 'Guangdong Provincial Key Laboratory of \\ Malignant Tumor Epigenetics and Gene \\ Regulation, Medical Research Center, Sun \\ Yat-Sen Memorial Hospital, Sun Yat-Sen \\ University, Guangzhou, People's Republic \\ of China; ${ }^{2}$ RNA Biomedical Institute, Sun \\ Yat-Sen Memorial Hospital, Sun Yat-Sen \\ University, Guangzhou, People's Republic \\ of China; ${ }^{3}$ Department of Radiation \\ Oncology, Sun Yat-Sen Memorial \\ Hospital, Sun Yat-Sen University, \\ Guangzhou, People's Republic of China; \\ ${ }^{4}$ Department of Oncology, Sun Yat-Sen \\ Memorial Hospital, Sun Yat-Sen \\ University, Guangzhou, People's Republic \\ of China; ${ }^{5}$ Breast Tumor Center, Sun Yat- \\ Sen Memorial Hospital, Sun Yat-Sen \\ University, Guangzhou, People's Republic \\ of China
}

Background: Due to the low rate of regional recurrence (RR) in early-stage breast cancer with pT1-2 and negative sentinel lymph node biopsy (SLNB), no regional therapy is suggested for them. However, whether there is a subset of patients who were with high risk of regional failure and may benefit from regional treatment is still unknown. The current study was designed to identify the patients with high risk of RR, thereby providing clues for enhanced regional therapy.

Methods: We analyzed a total of 1124 breast cancer patients with pT1-2N0 from May 2004 to Dec 2014. All the patients were treated with breast-conservation surgery (BCS) and adjuvant whole-breast radiotherapy. The regional recurrence-free survival (RRFS), local regional recurrence-free survival (LRRFS), disease-free survival (DFS) and overall survival (OS) were assessed by using the Kaplan-Meier method. Cox proportional hazards regression was performed to detect factors in predicting the RRFS.

Results: In multivariable analysis, both $\mathrm{T}$ stage and molecular type were significant predictors of RRFS. Patients with T2 stage had a lower RRFS than those with T1stage. Triplenegative patients were more likely to suffer regional failure than the patients with other molecular types. The two predictors were then employed to divide all the patients into three groups based on the risk level of RR. Patients with both T2 and triple-negative molecular type had the lower RRFS, LRRFS, DFS and OS than the patients with one or no risk factor. Conclusion: For early-stage breast cancer patients with negative SLNB, those who were with both T2 stage and triple-negative molecular type had a high rate of RR and enhance regional therapy may be needed for them.

Keywords: breast cancer, regional recurrence, radiotherapy

\section{Introduction}

Breast cancer is one of the most common malignant tumors in women, which causes approximately 626,679 deaths per year. ${ }^{1}$ Treatment strategy of breast cancer depends on the tumor stage. In patients with early-stage, breast-conserving surgery (BCS) followed by postoperative radiotherapy is considered as the standard care., ${ }^{2,3}$ When radiotherapy is used, usually, the radiation filed includes only the whole breast in T1-2 patients with negative lymph node metastasis excluding the regional nodal from radiation. In addition, the NCCN guideline also recommends that there is no need to irradiate the lymphatic drainage area in patients with pT1-2N0. ${ }^{4}$ Thus, in patients with pT1-2 and negative sentinel lymph node biopsy (SLNB), the regional nodal area is left "untreated". However, in the clinical practice, there is
Correspondence: He-rui Yao; Yi-min Liu Tel +86-20-8I 332533

Fax +86-20-8133253

Email yaoherui@mail.sysu.edu.cn;

liuyim@mail.sysu.edu.cn 
still a small number of patients who develop regional recurrence (RR). ${ }^{5-7}$ Whether there is a subset of these patients who have high risk of RR and may benefit from regional therapy such as radiation, is still unknown. Our current aimed to explore the RR rate in early-stage breast cancer treated with BCS plus radiotherapy. Furthermore, we also aimed to identify the subset of patients with a high risk of regional failure. This study will provide further evidence for enhanced regional treatment such as radiotherapy or surgery in patients with high RR rate.

\section{Methods and Materials}

\section{Ethics Statement}

The research permission from the Institutional Review Board of Sun Yat-Sen Memorial Hospital was achieved in this study. Besides, in our hospital, there was no need for written consent due to the retrospective property. We confirmed that we have protected the confidentiality of patients and would not release any private information of the patients to the public.

\section{Patients}

We used a database with medical records of patients treated at our hospital to obtain the relevant information. We selected the patients who underwent breast-conserving surgery between May 2004 and Dec 2014. In addition, the baseline characteristics such as age, tumor stage, pathologic reports, adjuvant therapy, and follow-up data had to be available. Specifically, the selection criteria for the current study were as follows: (1) pathologically confirmed invasive breast cancer with the stage of pT1-2N0; (2) underwent breast-conserving surgery (all achieved negative margins); (3) with negative SLNB; (3) received adjuvant radiotherapy for the whole breast. Patients who received neoadjuvant chemotherapy or had special pathologic types were not included. Furthermore, the patients who underwent axillary lymph node dissection (ALND) were also excluded with the aim to minimize the heterogeneity in patient selection. Finally, 1124 patients met the criteria and were included for the present analysis.

\section{Treatment}

Breast-conserving surgery was conducted in each patient and a negative surgical margin proved by pathology was achieved in all. After the completion of surgery, all patients underwent adjuvant radiotherapy, for which 6 or $10-\mathrm{MV}$ photons were commonly used to execute the radiation therapy. Usually, the prescription dose for the whole breast was $50 \mathrm{~Gy} / 25 \mathrm{~F}$. When the whole breast reached the total dose of $50 \mathrm{~Gy}$, a boost of tumor bed was then performed with a dose ranging from $10 \mathrm{~Gy}$ in 5 fractions to 16 Gy in 8 fractions. Radiation filed of the boost usually included the volume enclosed by surgical clips which were placed during the operation to identify the primary tumor position. Postoperative chemotherapy was administered to 942 patients and the regimens of chemotherapy were selected according to the NCCN guideline and the physicians' decision which was made based on the general condition of patients and their tumor stages. A targeted therapy, such as trastuzumab was suggested, especially to the patients with HER2 positive tumors. In patients with estrogen receptor (ER) and/or progesterone receptor (PR) positive tumors, endocrine therapy was also recommended with the aim to improve the treatment efficacy.

\section{Pathologic Evaluation}

Estrogen (ER) and progesterone receptor (PR) statuses were evaluated using immunohistochemistry (IHC) and the staining of IHC was read by specialized pathologists. The definitions of positive of ER and PR status were according to the well-established cut-off values (1\% for ER and $20 \%$ for PR). HER2 positive status was defined as a staining score over 3 . However, if the staining score for HER2 was 2, fluorescence in situ hybridization was then performed with HER2 gene amplification considered positive. Molecular type was assessed according to the St Gallen International Breast Cancer Conference (2013) Expert Panel. All the patients were divided into four groups as luminal A, luminal B, HER2-enriched and triple-negative breast cancer.

\section{Follow-Up Evaluation}

After the completion of treatment, the patients were suggested to be evaluated every 3 months for the first 2 years. Then, the time interval between two visits could be extended to 6 months in the following 3 years. After the patients have been followed up for 5 years, they could just visit the physician annually. Routine evaluations included blood tests, physical examinations and color doppler ultrasonography. Mammography and computed tomography were generally performed annually. Once recurrent signs were found in a patient, the biopsy was further performed to confirm that. Regional recurrence (RR) was defined as recurrence occurred only in lymphatic drainage areas 
which included the axillary, internal mammary, infraclavicular and supraclavicular lymph nodes. Loco-regional recurrence was defined as recurrence developed in ipsilateral breast or regional lymphatic drainage areas.

\section{Statistical Analysis}

We performed all the statistical analyses by using SPSS software, version 19.0. The chi-square test or Fisher's exact test was used for analyzing categorical variables. Continuous variables were analyzed using the Student's $t$-test or the Mann-Whitney $U$-test. The cox proportional hazards regression was employed to perform the multivariate analysis. All the possible clinical factors were included in the cox proportional hazards model by using a forward conditional selection of variables. In addition, the survival analysis was done by using the Kaplan-Meier method and the difference between the patient group was compared using the log rank test. In our study, the primary endpoint was regional recurrence-free survival (RRFS). And the secondary endpoints were loco-regional recurrence free survival (LRRFS), disease-free survival (DFS) and overall survival (OS). $\mathrm{P}<0.05$ was considered to indicate statistically significance.

\section{Results}

\section{Clinical Characteristics}

In total, 1124 patients were enrolled in the present study. There were 505 (44.9\%) patients who were younger than 45 years and the other 619 (55.1\%) patients were older than 45 years. T1 stage was diagnosed in 796 patients and the other 328 patients had T2 stage. In all of the included patients, the molecular subtype of luminal-A, luminal-B, HER2-enriched and triple-negative breast cancer were 685 (60.9\%), 190 (16.9\%), 84 (7.5\%) and 165 (14.7\%), respectively. Lymphovascular invasion (LVI) was detected in $120(10.6 \%)$ patients. Most patients received chemotherapy, but $182(16.2 \%)$ did not. Endocrine therapy was administered to 773 patients and the other 351 failed to receive it due to some reasons such as no indication or refusal (Table 1).

\section{Regional Control and Survival Analysis in All Patients}

During the follow-up, there were 54 patients who died and the 10-year OS rate for the whole group was $92.5 \%$. Recurrence was found in 94 patients, with 10-year DFS rates of $88.9 \%$. Among patients with recurrence, 34 patients
Table I Baseline Characteristics of the Patients

\begin{tabular}{|c|c|c|}
\hline Variables & Number & Percent (\%) \\
\hline \multicolumn{3}{|l|}{ Age, year } \\
\hline$\leq 45$ & 505 & $44.9 \%$ \\
\hline$>45$ & 619 & $55.1 \%$ \\
\hline \multicolumn{3}{|l|}{ Histologic type } \\
\hline Invasive ductal carcinoma & 998 & $88.8 \%$ \\
\hline Invasive lobular carcinoma & 34 & $3.0 \%$ \\
\hline Invasive papillary carcinoma & 37 & $3.2 \%$ \\
\hline Mucinous adenocarcinoma & 55 & $5.0 \%$ \\
\hline \multicolumn{3}{|l|}{ PT stage } \\
\hline $\mathrm{TI}$ & 796 & $70.8 \%$ \\
\hline $\mathrm{T} 2$ & 328 & $29.2 \%$ \\
\hline \multicolumn{3}{|l|}{ ER status } \\
\hline Positive & 868 & $77.2 \%$ \\
\hline Negative & 256 & $22.8 \%$ \\
\hline \multicolumn{3}{|l|}{ PR status } \\
\hline Positive & 807 & $71.8 \%$ \\
\hline Negative & 317 & $28.2 \%$ \\
\hline \multicolumn{3}{|l|}{ Her2 status } \\
\hline Positive & 173 & $15.4 \%$ \\
\hline Negative & 951 & $84.6 \%$ \\
\hline \multicolumn{3}{|l|}{ Molecular type } \\
\hline Luminal A & 685 & $60.9 \%$ \\
\hline Luminal B & 190 & $16.9 \%$ \\
\hline Her2-enriched & 84 & $7.5 \%$ \\
\hline Triple negative & 165 & $14.7 \%$ \\
\hline \multicolumn{3}{|l|}{ LVI } \\
\hline Yes & 120 & $10.7 \%$ \\
\hline No & 1004 & $89.3 \%$ \\
\hline \multicolumn{3}{|l|}{ Chemotherapy } \\
\hline Yes & 942 & $83.8 \%$ \\
\hline No & 182 & $16.2 \%$ \\
\hline \multicolumn{3}{|l|}{ Endocrine therapy } \\
\hline Yes & 773 & $68.8 \%$ \\
\hline No & 351 & $31.2 \%$ \\
\hline
\end{tabular}

Abbreviation: LVI, lymphovascular invasion.

developed local-regional recurrence and 41 patients presented with only distant metastasis. Additionally, 19 patients developed both local-regional and distant failures. RR was found in 18 patients and the sites of regional failure were supraclavicular and axillary areas. In detail, 3 patients were with supraclavicular region recurrence, 14 recurred within the area of axillary lymphatic drainage and 1 suffered both supraclavicular and axillary recurrence. The 10 -year RR rate was $2.4 \%$ for all the patients. 


\section{Clinical Predictors for Regional}

\section{Recurrence in the Whole Group}

In univariate analysis, tumor stage and molecular type could predict the RR (Table 2). The other factors such as age, tumor grade, LVI, adjuvant chemotherapy and endocrine therapy were not significantly associated with RRFS. Multivariable analysis was further performed and the results showed that both the tumor stage and molecular type were still independent predictors of RRFS (Table 2). Thereby, T2 patients had a 4.5-fold risk to develop RR compared to those with T1stage. Furthermore, triplenegative patients also had a higher tendency to suffer regional failure than those with other molecular types.

\section{Subgroup Analysis Based on Factor of T Stage}

Based on the results of multivariable analysis, we further analyze the survival data in different subgroup. In patients with T1 stage, there were 45 patients who recurred. Fourteen patients recurred only local-regionally and 24 patients developed only distant metastasis. Seven patients experienced both local-regional and distant failures. RR was found in six cases. The 10-year RRFS, LRRFS, and DFS rates were $99.16 \%, 96.40 \%$ and $92.64 \%$, respectively. In patients with T2 stage, 49 patients developed recurrence. Isolated local-regional recurrence was observed in
20 patients and isolated distant metastasis was found in 17 patients. There were 12 patients who developed both localregional and distant failures. RR was observed in 12 patients. The 10-year RRFS, LRRFS and DFS rates were 93.51\%, 85.77\% and 79.36\%, respectively. Compared to patients with T1 stage, those with T2 tumors acquired a poorer RRFS, LRRFR and DFS (Table 3 and Figure 1).

\section{Subgroup Analysis Based on Factor of Molecular Type}

In patients with triple-negative molecular type, seven patients developed RR. As for patients with non-triplenegative molecular type, there were 11 patients who showed with RR. The 10-year RRFS for the two groups were $94.87 \%$ and $98.07 \%$, respectively. Triple-negative patients had a significantly poorer RRFS than those with other molecular types. Furthermore, the patients in the triple-negative group also had a lower LRRFS, DFS and OS than those in the nontriple-negative group (Table 4 and Figure 2).

\section{Combined Analysis with T Stage and Molecular Type in Defining Risk Group for Regional Recurrence}

We adopted the two risk factors to divide all the patients into three groups based on the risk level of RR. The high-risk group included patients with both T2 stage and triple-negative

Table 2 Univariate and Multivariate Analyses of Regional Recurrence

\begin{tabular}{|c|c|c|c|c|}
\hline \multirow[t]{2}{*}{ Variables } & \multicolumn{2}{|c|}{ Univariate Analysis } & \multicolumn{2}{|c|}{ Multivariate Analysis } \\
\hline & HR $(95 \% \mathrm{CI})$ & p value & HR (95\% Cl) & p value \\
\hline \multicolumn{5}{|l|}{ T stage } \\
\hline TI $(n=796)$ vs T2 $(n=328)$ & $0.20(0.07-0.53)$ & 0.001 & $0.22(0.08-0.60)$ & 0.003 \\
\hline \multicolumn{5}{|l|}{ Tumor grade } \\
\hline I-II $(n=635)$ vs III $(n=489)$ & $0.92(0.36-2.33)$ & 0.862 & & \\
\hline \multicolumn{5}{|l|}{ LVI } \\
\hline No $(n=1004)$ vs yes $(n=120)$ & $0.47(0.14-1.64)$ & 0.238 & & \\
\hline \multicolumn{5}{|l|}{ Molecular type } \\
\hline Triple-negative $(n=166)$ vs non-triple-negative $(n=958)$ & $3.83(1.49-9.89)$ & 0.005 & $3.32(1.21-8.16)$ & 0.019 \\
\hline \multicolumn{5}{|l|}{ Age } \\
\hline$\leq 45(n=505)$ vs $>45(n=619)$ & $1.89(0.73-4.87)$ & 0.189 & & \\
\hline \multicolumn{5}{|l|}{ Chemotherapy } \\
\hline No $(n=182)$ vs Yes $(n=942)$ & $1.86(0.61-5.70)$ & 0.278 & & \\
\hline \multicolumn{5}{|l|}{ Endocrine therapy } \\
\hline No $(n=35 I)$ vs Yes $(n=773)$ & I.8I (0.7I-4.59) & 0.212 & & \\
\hline
\end{tabular}

Abbreviations: $\mathrm{Cl}$, confidence interval; $\mathrm{HR}$, hazard ratio; LVI, lymphovascular invasion. 
Table 3 Subgroup Analysis Based on T Stage

\begin{tabular}{|l|l|l|l|l|l|}
\hline \multirow{2}{*}{ Group } & \multicolumn{2}{|l|}{$\begin{array}{l}\text { pTI Subgroup } \\
\text { (N=796) }\end{array}$} & \multicolumn{2}{l|}{$\begin{array}{l}\text { pT2 Subgroup } \\
\mathbf{( N = 3 2 8 )}\end{array}$} & \multirow{2}{*}{ P value } \\
\cline { 2 - 5 } & $\mathbf{5 - Y e a r}$ & $10-$ Year & 5-Year & 10-Year & \\
\hline RRFS & $99.16 \%$ & $99.16 \%$ & $97.17 \%$ & $93.51 \%$ & $<0.001$ \\
LRRFS & $97.71 \%$ & $96.40 \%$ & $92.21 \%$ & $85.77 \%$ & $<0.001$ \\
DFS & $94.68 \%$ & $92.64 \%$ & $88.11 \%$ & $79.36 \%$ & $<0.001$ \\
OS & $97.60 \%$ & $93.41 \%$ & $95.88 \%$ & $90.28 \%$ & 0.033 \\
\hline
\end{tabular}

Abbreviations: RRFS, regional recurrence-free survival; LRRFS, local regiona recurrence-free survival; DFS, disease-free survival; OS, overall survival.

molecular type. Patients with either T2 or triple-negative type were in the intermediate-risk group. Low-risk patients were those with both $\mathrm{T} 1$ stage and non-triple-negative molecular type. Survival data were analyzed and the RRFS for the high-, intermediate-, and low-risk groups were $92.35 \%, 95.01 \%$ and $99.50 \%$, respectively. There was a significant difference in RRFS between the three groups $(\mathrm{P}<0.001)$. Furthermore, the LRFS, DFS and OS in the high-risk groups were also found to be lower than that in the intermediate and low-risk groups (Table 5 and Figure 3).
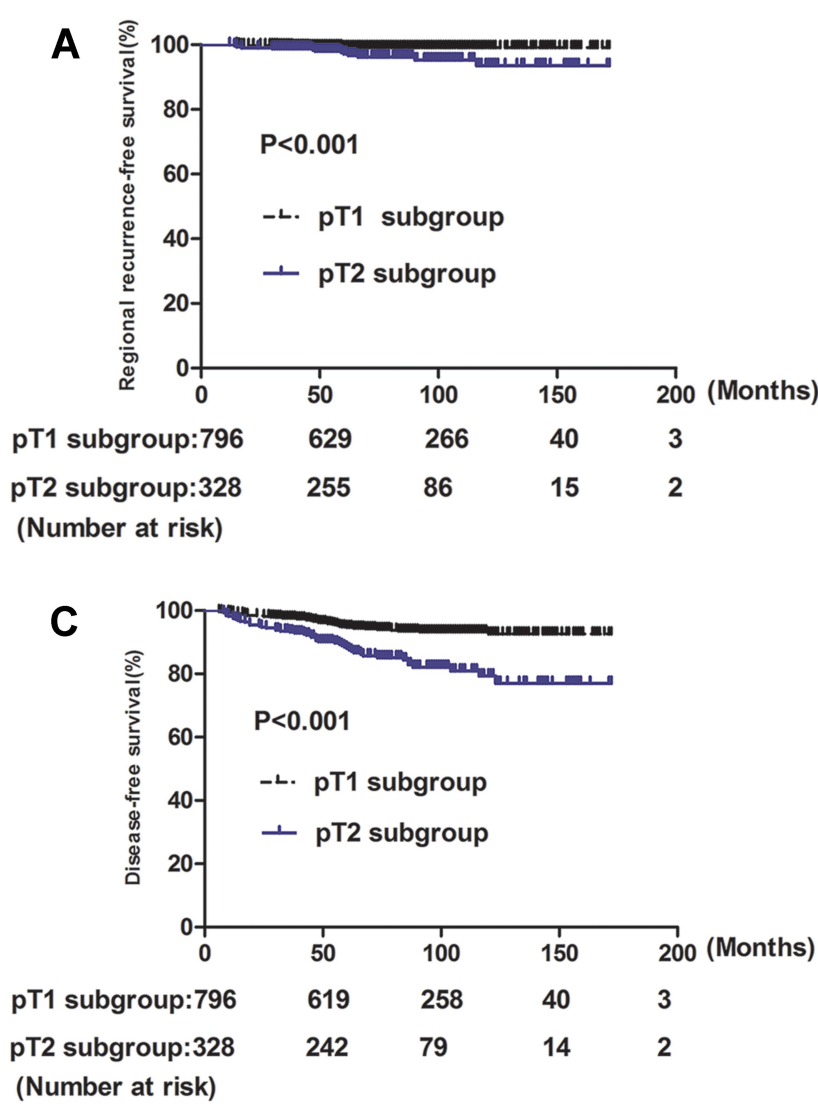

\section{Discussion}

The primary goal of the current study was to evaluate the RR rate in early-stage breast cancer patients. Our results showed a 10 -year RR rate of $2.4 \%$ with a 10 -year DFS of $88.9 \%$, which was consistent with previously published studies. ${ }^{5,6}$ As we know, for early-stage breast cancer with pT1-2N0, the radiation field suggested by $\mathrm{NCCN}$ guideline includes only the whole breast, while it is not essential to irradiate the regional nodal area. ${ }^{4}$ Thus, regional area was left "untreated" in patients with negative SLNB and no further ALND. However, we were still not sure whether there was a subset of patients who may need regional treatment such as radiotherapy due to their high risk of RR. To address this clinical problem, we firstly explored which clinical factors were significantly associated with RR. The results showed that pT stage and molecular type of the tumor were significant predictors of RR. Compared to patients with T1 stage, those with T2 stage suffered a 4.5 -fold risk of RR. In addition, triple-negative patients were also more likely to develop RR than those with other molecular types. We then used the two factors of $\mathrm{T}$ stage and molecular type to divide all the patients

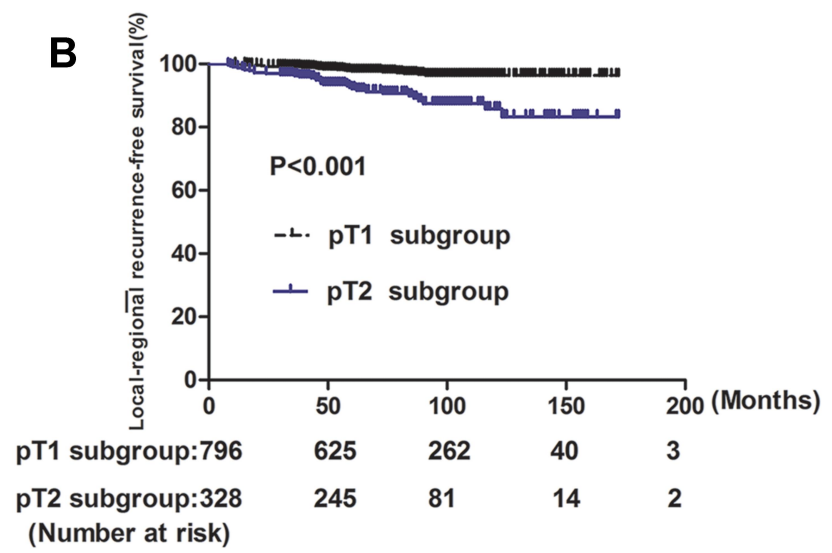

\section{D}

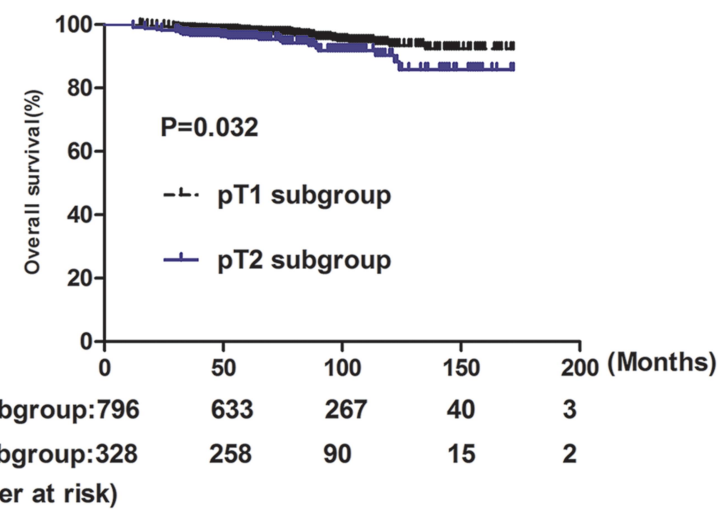

Figure I The survival analysis based on T stage. (A) regional recurrence free-survival (RRFS); (B) local regional recurrence-free survival (LRRFS); (C) disease-free survival (DFS); (D) overall survival (OS). Patients with TI stage had a significantly higher RRFS, LRRFS, DFS and OS than patients with $\mathrm{T} 2$ stage $(\mathrm{P}<0.05)$. 
Table 4 Subgroup Analysis Based on Molecular Subtype

\begin{tabular}{|l|l|l|l|l|l|}
\hline \multirow{2}{*}{ Group } & \multicolumn{2}{|l|}{$\begin{array}{l}\text { Triple-Negative } \\
\text { Subgroup (N=166) }\end{array}$} & \multicolumn{2}{l|}{$\begin{array}{l}\text { Non-Triple- } \\
\text { Negative Subgroup } \\
\text { (N=958) }\end{array}$} & \\
& \multicolumn{2}{|l|}{ P value } \\
\cline { 2 - 5 } & $\mathbf{5 - Y e a r}$ & $10-Y e a r$ & $\mathbf{5 - Y e a r}$ & $10-Y e a r$ & \\
\hline RRFS & $95.78 \%$ & $94.87 \%$ & $99.05 \%$ & $98.07 \%$ & 0.003 \\
LRRFS & $92.09 \%$ & $89.92 \%$ & $96.79 \%$ & $94.00 \%$ & 0.011 \\
DFS & $87.36 \%$ & $84.39 \%$ & $93.69 \%$ & $89.66 \%$ & 0.004 \\
OS & $92.42 \%$ & $88.25 \%$ & $97.91 \%$ & $93.26 \%$ & 0.002 \\
\hline
\end{tabular}

Abbreviations: RRFS, regional recurrence-free survival; LRRFS, local regional recurrence-free survival; DFS, disease-free survival; OS, overall survival.

into three groups based on different risk level of RR. We found that patients with both T2 stage and triple-negative molecular type had the higher risk of RR than those with only one risk factor or no risk factor. Furthermore, the patients in the high-risk group also presented with a poorer DFS and OS than those in the low- or medium-risk groups. Thereby, we suggested that enhanced regional treatment may be needed for triple-negative breast cancer with $\mathrm{T} 2$ stage.

Several studies have investigated the problem of regional nodal failure in breast cancer patients. ${ }^{5-9}$ For example, Grills et al performed a study that included 1500 cases of Stage I-II breast cancer treated with breast-conserving therapy. They reported that the 5- and 10-year rate for RR was $1.9 \%$ and $2.8 \%$, respectively, which was slightly higher than that reported by us. The reason for this discrepancy was that Grills et al had enrolled the patients with positive lymph node metastasis. Their data also indicated that regional radiation was useful for patients with more than three positive lymph nodes because it significantly reduced the rate of RR. ${ }^{5}$ In another study reported by Wo et al, 1000 patients were retrospectively analyzed and there were only 6 patients who have developed isolated RR. ${ }^{7}$ They also found that tumor biological subtype was associated with higher RR rate, which was in line with our findings. Recently, a large populationbased study by van Steenhoven et $\mathrm{al}^{10}$ assessed early-stage (N0) breast cancer patients and found that the use of wholebreast radiotherapy was associated with a lower risk of RR, while the younger age, larger tumor size, and higher grade were associated with higher RR rate. Their results were partly consistent with our findings and we also found that patients with large tumors were more likely to develop RR than those with small tumors. Although our study shared several common points with the study of van Steenhoven et al, there were significant differences between the two.
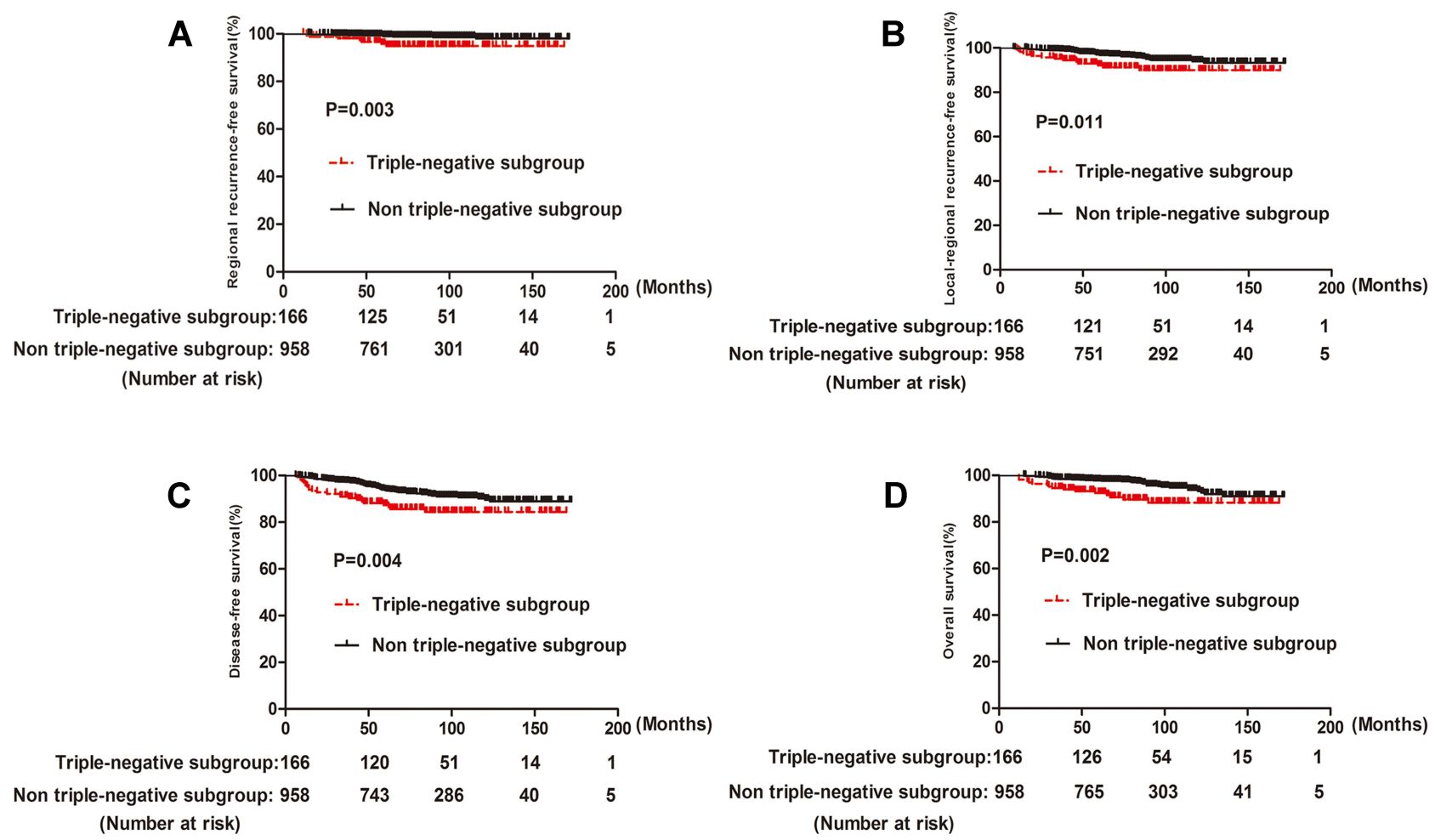

Figure 2 The survival analysis based on molecular subtype. (A) regional recurrence free-survival (RRFS); (B) local regional recurrence-free survival (LRRFS); (C) diseasefree survival (DFS); (D) overall survival (OS). Patients with triple-negative molecular type had a significantly lower RRFS, LRRFS, DFS and OS than patients with other molecular types $(\mathrm{P}<0.05)$. 
Table 5 Combined Analysis of Molecular Subtype and PT Stage

\begin{tabular}{|l|l|l|l|l|l|l|l|}
\hline \multirow{2}{*}{ Group } & \multicolumn{2}{|l|}{ High-Risk Group (N=70) } & \multicolumn{2}{l|}{ Intermediate-Risk Group (N=354) } & \multicolumn{2}{l|}{ Low-Risk Group (N=700) } & P value \\
\cline { 2 - 8 } & $\mathbf{5 - Y e a r}$ & $\mathbf{1 0 - Y e a r}$ & $\mathbf{5 - Y e a r}$ & $\mathbf{1 0 - Y e a r}$ & $\mathbf{5}$-Year & $\mathbf{1 0}$-Year \\
\hline RRFS & $94.66 \%$ & $92.35 \%$ & $97.50 \%$ & $95.01 \%$ & $99.50 \%$ & $99.50 \%$ & $<0.001$ \\
LRRFS & $88.74 \%$ & $82.80 \%$ & $93.48 \%$ & $89.21 \%$ & $98.14 \%$ & $96.62 \%$ & $<0.001$ \\
DFS & $83.31 \%$ & $75.16 \%$ & $89.64 \%$ & $83.84 \%$ & $95.27 \%$ & $92.87 \%$ & $<0.001$ \\
OS & $92.86 \%$ & $83.48 \%$ & $95.45 \%$ & $92.01 \%$ & $98.36 \%$ & $93.66 \%$ & 0.001 \\
\hline
\end{tabular}

Abbreviations: RRFS, regional recurrence-free survival; LRRFS, local regional recurrence-free survival; DFS, disease-free survival; OS, overall survival.

First, the patients enrolled in our study were all staged as pT1-2N0 and were treated with BCS followed by wholebreast radiation. There was a portion of patients who received mastectomy in the study of van Steenhoven et al and that may partly explain the different findings between the two studies. Secondly, the goal of our study was to identify the patients who were with a high risk of RR, thereby suggesting that enhanced regional therapy such as radiotherapy may be needed for these patients.

There was another study which was performed by Hirata who reported the RR rate in patients treated by breastconserving surgery without regional radiotherapy. ${ }^{11}$ All of the included patients were with positive lymph nodes and the 5- and 10-year RRFS rates were $97.4 \%$ and $93.7 \%$, respectively. The patients in our study also did not receive regional radiotherapy. However, we noticed that the RRFS rate in the high-risk groups $(92.35 \%)$ was slightly lower than that reported by Hirata et al. This may partly have indicated that, in patients with $\mathrm{N} 0$, there was still a subset of patients who suffered higher RR rate than patients with positive lymph nodes. Our study has identified two independent risk factors associated with RR and we found that patients with both the two risk factors showed a significantly higher propensity to suffer RR than those with no risk factors. Perhaps,

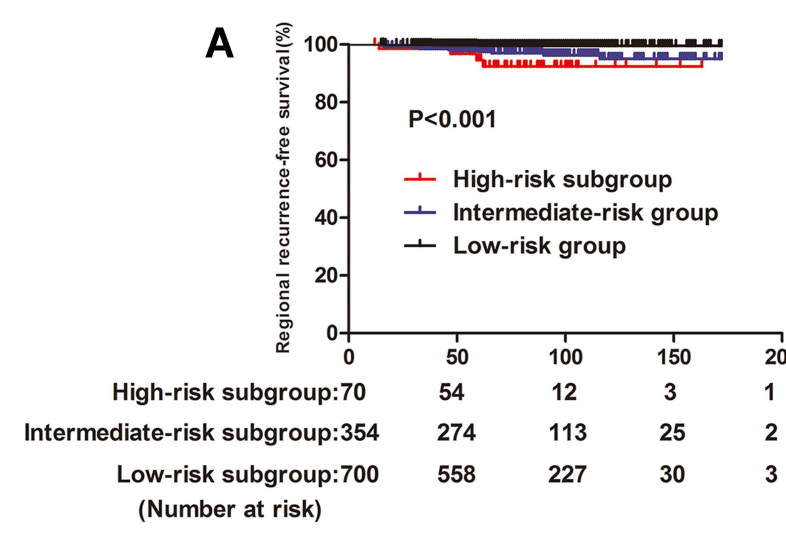

C

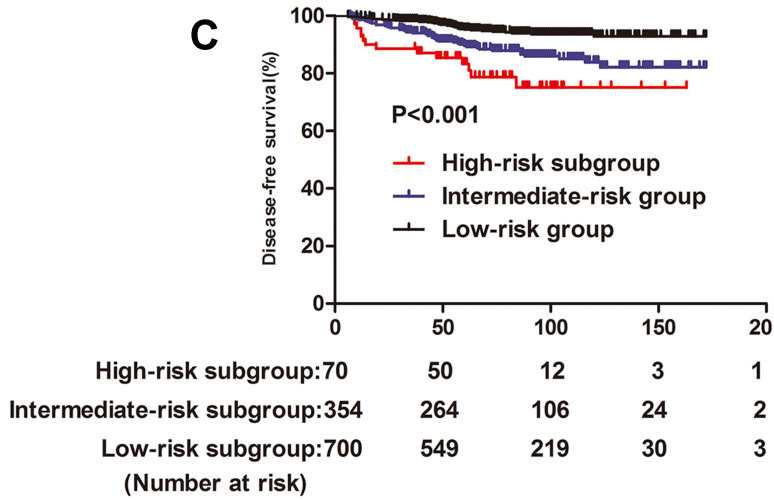

(1)

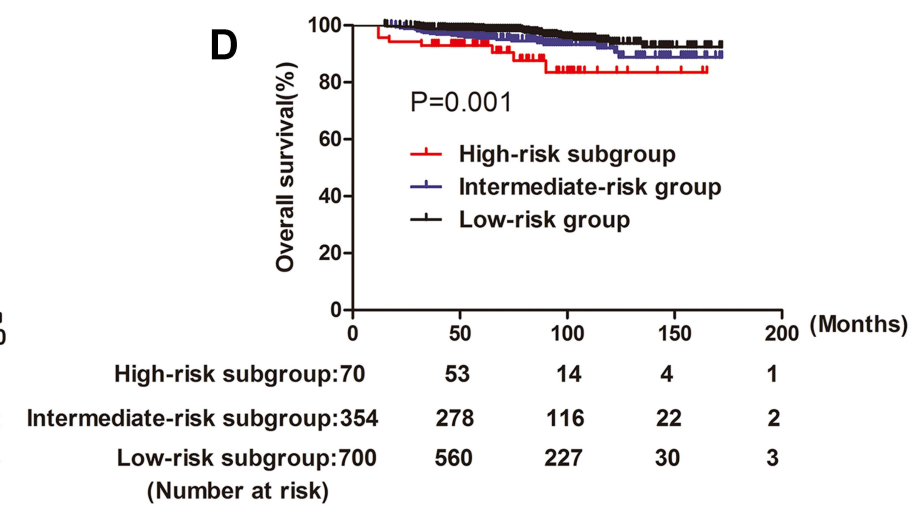

Figure 3 The survival analysis based on risk level of RR. (A) regional recurrence free-survival (RRFS); (B) local regional recurrence-free survival (LRRFS); (C) disease-free survival (DFS); (D) overall survival (OS). Patients in the high-risk group had poorer RRFS, LRRFS, DFS and OS than patients in the intermediate and low-risk groups (P<0.05). 
enhanced regional therapy such as radiotherapy could be selectively given to these patients.

Indeed, regional nodal irradiation has been found to improve DFS in patients with early-stage breast cancer. Data from a large randomized controlled trial showed that patients who received regional radiation had better DFS than those who did not. ${ }^{12}$ Similar conclusions were also drawn in the trial of DBCG $82 \mathrm{~b}$ and $\mathrm{c}$ which enrolled early-stage breast cancer patients and showed that regional radiotherapy was associated with increased DFS. ${ }^{13}$ Although the two trials have included patients with positive lymph nodes in the analysis, it may be reasonable to suppose that patients in the high-risk group defined in our study may also benefit from regional radiation due to their relatively high rate of $\mathrm{RR}$. A large clinical trial may be needed to evaluate the efficacy of regional radiotherapy in pT1-2N0 patients with high risk of RR.

Our study also demonstrated that molecular and tumor stage were independent predictors of poor LRRFS and DFS, which was in line with other studies. ${ }^{14-18}$ It has been reported that triple-negative patients had a significantly higher rate of LRR than those with other molecular types. ${ }^{14-16}$ Additionally, large tumor size was also found to be associated with impaired tumor prognosis. ${ }^{17,18}$ In our current study, by combining tumor size and molecular type in the analysis, we have defined a subgroup of patients who were with high risk of regional recurrence in early-stage breast cancer with negative SLNB. And the rate of RR for high-risk patients was $7.7 \%$, which was higher than those with only one or no risk factor. Since no regional therapy was administered to patients with negative SLNB, those with both large tumor size and triple-negative molecular type may benefit from additional regional therapy or enhanced observation of regional lymphatic drainage areas. However, a large clinical trial was still warranted to confirm our finding. Besides, large tumor size combined by triple-negative molecular type also indicated a poor prognosis. We noted that patients with both triple-negative molecular type and T2 stage had the worst DFS and OS as well.

This study has several limitations. First, it was a retrospective design study and we could not exclude the possibility of the selection bias. Second, since we have divided the patients into three groups based on the risk factors of RR and the sample size may have been small in some subgroups. However, our study is the first one to identify the patients with high risk of $R R$ in the early-stage breast cancer with negative SLNB and this study will help the physicians to make the tailored regional treatment for these patients.

\section{Conclusion}

In conclusion, among the patients with T1-2 stage and negative SLNB, those with both triple-negative molecular type and $\mathrm{T} 2$ tumor displayed a relatively high $\mathrm{RR}$ rate. We suggest that enhanced regional therapy may be needed for these patients. However, a large clinical trial is warranted to confirm our hypothesis.

\section{Abbreviations}

$\mathrm{RR}$, regional recurrence; RRFS, regional recurrence-free survival; LRRFS, local regional recurrence-free survival; DFS, disease-free survival; OS, overall survival; CI, confidence interval; HR, hazard ratio; LVI, lymphovascular invasion; BCS, breast-conservation surgery; SLNB, sentinel lymph node biopsy; ALND, axillary lymph node dissection.

\section{Funding}

This study was supported by grants from the National Science and Technology Major Project (2020ZX09201021), the National Natural Science Foundation of China (81572596, 81972471, U1601223), the Natural Science Foundation of Guangdong Province (2017A030313828, 2016A030313312), the Guangzhou Science and Technology Major Program (201704020131), the Sun YatSen University Clinical Research 5010 Program (2018007), the Sun Yat-Sen Clinical Research Cultivating Program (SYS-C-201801), and the grant from Guangdong Science and Technology Department (2017B030314026).

\section{Disclosure}

Kai-yun You, Zhuo-fei Bi, and Lin Ding are equal contributors. The authors declare that they have no competing interests for this work.

\section{References}

1. Bray F, Ferlay J, Soerjomataram I, et al. Global cancer statistics 2018: GLOBOCAN estimates of incidence and mortality worldwide for 36 cancers in 185 countries. CA Cancer J Clin. 2018;68(6):394-424. doi:10.3322/caac. 21492

2. Fisher B, Bryant J, Dignam JJ, et al. Tamoxifen, radiation therapy, or both for prevention of ipsilateral breast tumor recurrence after lumpectomy in women with invasive breast cancers of one centimeter or less. J Clin Oncol. 2002;20(20):4141-4149. doi:10.1200/JCO.2002.11.101

3. Early breast cancer trialists' collaborative group. Effect of radiotherapy after breast-conserving surgery on 10-year recurrence and 15 -year breast cancer death: meta-analysis of individual patient data for 10,801 women in 17 randomised trials. Lancet. 2011;378(9804):1707-1716. doi:10.1016/S0140-6736(11)61629-2 
4. National Comprehensive Cancer Network. NCCN clinical practice guidelines in oncology_breast cancer; 2019. Available from: http:// www.nccn.org/. Accessed September 12, 2020.

5. Grills IS, Kestin LL, Goldstein N, et al. Risk factors for regional nodal failure after breast-conserving therapy: regional nodal irradiation reduces rate of axillary failure in patients with four or more positive lymph nodes. Int J Radiat Oncol Biol Phys. 2003;56 (3):658-670. doi:10.1016/S0360-3016(03)00017-8

6. Truong PT, Jones SO, Kader HA, et al. Patients with t1 to t2 breast cancer with one to three positive nodes have higher local and regional recurrence risks compared with node-negative patients after breast-conserving surgery and whole-breast radiotherapy. Int J Radiat Oncol Biol Phys. 2009;73(2):357-364. doi:10.1016/j. ijrobp.2008.04.034

7. Wo JY, Taghian AG, Nguyen PL, et al. The association between biological subtype and isolated regional nodal failure after breast-conserving therapy. Int J Radiat Oncol Biol Phys. 2010;77 (1):188-196. doi:10.1016/j.ijrobp.2009.04.059

8. Wong JS, Recht A, Beard CJ, et al. Treatment outcome after tangential radiation therapy without axillary dissection in patients with early-stage breast cancer and clinically negative axillary nodes. Int J Radiat Oncol Biol Phys. 1997;39(4):915-920. doi:10.1016/ S0360-3016(97)00456-2

9. Boutrus R, Abi-Raad R, Niemierko A, et al. Does lymphovascular invasion predict regional nodal failure in breast cancer patients with zero to three positive lymph nodes treated with conserving surgery and radiotherapy? Implications for regional radiation. Int $J$ Radiat Oncol Biol Phys. 2010;78(3):793-798. doi:10.1016/j.ijrobp. 2009.08.049

10. van Steenhoven JEC, Kuijer A, van Maaren MC, et al. Quantifying the mitigating effects of whole-breast radiotherapy and systemic treatments on regional recurrence incidence among breast cancer patients [published online ahead of print, 2020 March 20]. Ann Surg Oncol. 2020;10. doi:1245/s10434-020-08356-2
11. Hirata K, Yoshimura M, Inoue M, et al. Regional recurrence in breast cancer patients with one to three positive axillary lymph nodes treated with breast-conserving surgery and whole breast irradiation. $J$ Radiat Res. 2017;58(1):79-85. doi:10.1093/jrr/rrw071

12. Poortmans PM, Collette S, Kirkove C, et al. Internal mammary and medial supraclavicular irradiation in breast cancer. $N$ Engl J Med. 2015;373(4):317-327. doi:10.1056/NEJMoa1415369

13. Overgaard M, Nielsen HM, Overgaard J. Is the benefit of postmastectomy irradiation limited to patients with four or more positive nodes, as recommended in international consensus reports? A subgroup analysis of the DBCG 82 b\&c randomized trials. Radiother Oncol. 2007;82 (3):247-253. doi:10.1016/j.radonc.2007.02.001

14. Tseng YD, Uno H, Hughes ME, et al. Biological subtype predicts risk of locoregional recurrence after mastectomy and impact of postmastectomy radiation in a large national database. Int J Radiat Oncol Biol Phys. 2015;93(3):622-630. doi:10.1016/j.ijrobp.2015.07.006

15. Yang TJ, Morrow M, Modi S, et al. The effect of molecular subtype and residual disease on locoregional recurrence in breast cancer patients treated with neoadjuvant chemotherapy and postmastectomy radiation. Ann Surg Oncol. 2015;22(Suppl S3):S495-S501. doi:10.1245/s10434-015-4697-7

16. Jwa E, Shin KH, Kim JY, et al. Locoregional recurrence by tumor biology in breast cancer patients after preoperative chemotherapy and breast conservation treatment. Cancer Res Treat. 2016;48(4):13631372. doi:10.4143/crt.2015.456

17. Ferguson NL, Bell J, Heidel R, et al. Prognostic value of breast cancer subtypes, Ki-67 proliferation index, age, and pathologic tumor characteristics on breast cancer survival in Caucasian women. Breast J. 2013;19(1):22-30. doi:10.1111/tbj.12059

18. Gómez-Acebo I, Dierssen-Sotos T, Palazuelos-Calderón C, et al. Tumour characteristics and survivorship in a cohort of breast cancer: the MCC-Spain study [published online ahead of print, 2020 April 30]. Breast Cancer Res Treat. 2020;10. doi:1007/s10549-020-05600-X

\section{Publish your work in this journal}

Cancer Management and Research is an international, peer-reviewed open access journal focusing on cancer research and the optimal use of preventative and integrated treatment interventions to achieve improved outcomes, enhanced survival and quality of life for the cancer patient.
The manuscript management system is completely online and includes a very quick and fair peer-review system, which is all easy to use. Visit http://www.dovepress.com/testimonials.php to read real quotes from published authors. 